\title{
Energy resolution and related charge carrier mobility in $\mathrm{LaBr}_{3}: \mathrm{Ce}$ scintillators
}

\author{
I. V. Khodyuk, F. G. A. Quarati, M. S. Alekhin, and P. Dorenbos \\ Luminescence Materials Research Group, Faculty of Applied Sciences, Delft University of Technology, \\ Mekelweg 15, Delft, 2629JB, The Netherlands
}

(Received 25 July 2013; accepted 12 September 2013; published online 27 September 2013)

\begin{abstract}
The scintillation response of $\mathrm{LaBr}_{3}: \mathrm{Ce}$ scintillation crystals was studied as function of temperature and $\mathrm{Ce}$ concentration with synchrotron $\mathrm{X}$-rays between $9 \mathrm{keV}$ and $100 \mathrm{keV}$. The results were analyzed using the theory of carrier transport in wide band gap semiconductors to gain new insights into charge carrier generation, diffusion, and capture mechanisms. Their influence on the efficiency of energy transfer and conversion from X-ray or $\gamma$-ray photon to optical photons and therefore on the energy resolution of lanthanum halide scintillators was studied. From this, we will propose that scattering of carriers by both the lattice phonons and by ionized impurities are key processes determining the temperature dependence of carrier mobility and ultimately the scintillation efficiency and energy resolution. When assuming about $100 \mathrm{ppm}$ ionized impurity concentration in $0.2 \% \mathrm{Ce}^{3+}$ doped $\mathrm{LaBr}_{3}$, mobilities are such that we can reproduce the observed temperature dependence of the energy resolution, and in particular, the minimum in resolution near room temperature is reproduced. (C) 2013 AIP Publishing LLC. [http://dx.doi.org/10.1063/1.4823737]
\end{abstract}

\section{INTRODUCTION}

The dynamics of hot charge carriers created in the ionization track of ionizing particles is of interest in various disciplines of science. In a small cylindrical volume with radius $\mathrm{r} \sim 5 \mathrm{~nm}$ around the ionization track ${ }^{1}$ schematically shown in Fig. 1 on the ps time scale ${ }^{2}$ a very high ionization density $\mathrm{n}>10^{20} \mathrm{e}-\mathrm{h} / \mathrm{cm}^{3}$ of free electrons and holes are created ${ }^{3,4}$ that can cause secondary effects. For instance the energy density available is sufficient to displace atoms from their normal lattice positions thus creating radiation damage. ${ }^{5}$ In tissue radiation damage may have severe health risks, and in dosimetry, it can lead to underestimation of the total absorbed dose. Currently, there are many investigations in utilizing carrier multiplication to develop better efficiency photo-voltaic cells. In inorganic scintillators, that is the topic of this work, the created free charge carriers need to escape the volume of high ionization density to be trapped by a luminescence center and recombine under emission of photons. ${ }^{6}$

Scintillation crystals are widely used as spectroscopic detectors of ionizing radiation in nuclear science, space exploration, medical imaging, homeland security, etc. The important parameters for X- or $\gamma$-ray spectrometry are the total light output by the scintillator expressed in photons emitted per $\mathrm{MeV}$ of absorbed ionizing energy, decay time of the scintillation light flash, energy resolution for the detection of the ionizing particle and the detection efficiency. Taking into account all parameters one of the best inorganic scintillator commercially available today is $\mathrm{LaBr}_{3}: \mathrm{Ce}^{7,8}$ Concerning high light output and good energy resolution the rediscovered ${ }^{9} \operatorname{SrI}_{2}: \mathrm{Eu}$ and recently discovered ${ }^{10} \mathrm{BaBrI}: \mathrm{Eu}$ and $\mathrm{CsBa}_{2} \mathrm{I}_{5}$ :Eu scintillators are very promising.

Physical processes following absorption of ionizing radiation by a scintillator and further energy conversion to optical photons are in the focus of the scintillation community for a long time. ${ }^{11,12}$ In spite of all the efforts, ${ }^{13-17}$ some aspects of the conversion mechanism are still unclear. For example, energy resolution of inorganic scintillators is still much larger than the fundamental limit dictated by photon statistics. ${ }^{18}$ Figure 2 shows the energy resolution achieved by well-known scintillators for the detection of $662 \mathrm{keV}$ gamma ray photons. The best resolution for commercially available scintillators is for $\mathrm{LaBr}_{3}$ :Ce followed by $\mathrm{SrI}_{2}:$ Eu. The star symbols are the fundamental limit as dictated by photon statistics for these two scintillators which demonstrates that there is still very significant improvement possible to well below $2 \%$. For a solid state detector like high purity germanium (HPGe) photon statistics does not contribute and much better resolution down to $0.3 \%$ can be obtained. To decrease the energy resolution by almost a factor of two to $1.8 \%$ for $\mathrm{LaBr}_{3}$ and to $1.5 \%$ for $\mathrm{SrI}_{2}$, it is necessary to minimize all contributions other than photon statistics that influence energy resolution. The most essential contribution to be minimized is the contribution determined by nonproportionality. ${ }^{19}$

Nonproportionality is the nonlinear dependence of the total light output of the scintillator on the absorbed amount of ionization energy, i.e., the emitted number of photons/ $\mathrm{MeV}$ at $10 \mathrm{keV}$ is not necessarily the same as at $100 \mathrm{keV}$ or at $1000 \mathrm{keV}$. This dependence is due to a scintillation efficiency that, in turn, depends on the density of the ionization track. The production of secondary electrons (i.e., Auger electrons, delta-rays, etc.) during slowing down of the primary electron is a probabilistic process and may occur in different ways for the same absorbed energy. The dependence of the absolute light yield on the energy of secondary electrons and the probabilistic mechanism of their creation result in variability of the total number of photons produced inside the scintillator. ${ }^{20}$ This process leads to broadening of the 


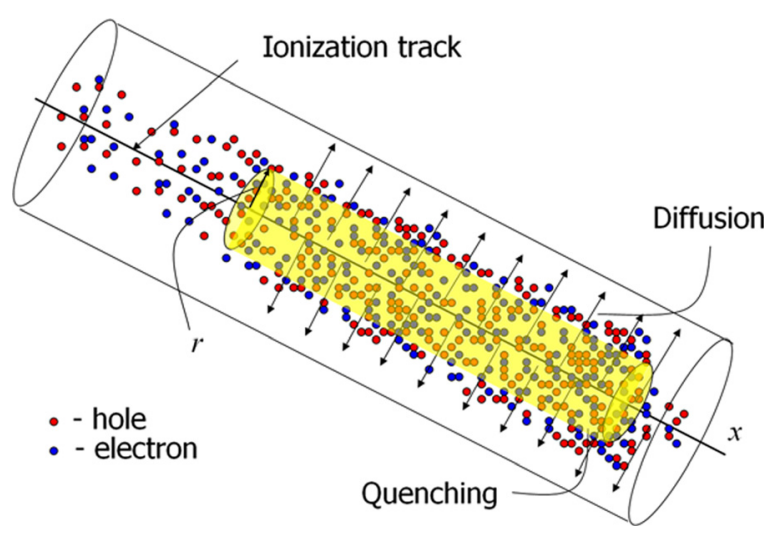

FIG. 1. Sketch of an ionization track formed by a primary electron starting from the left creating free electrons and holes that diffuse radially away from the track. Radiationless carrier recombination occurs at the dense carrier concentration regions.

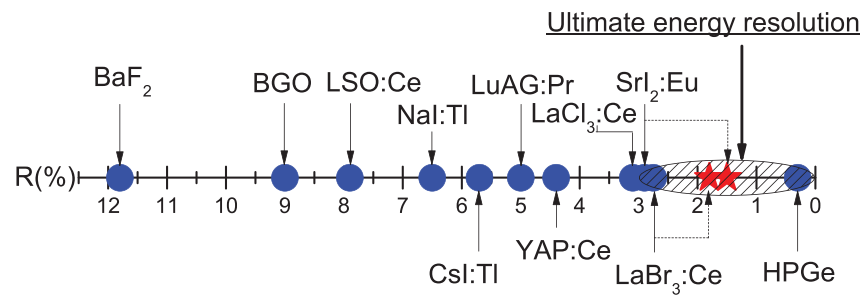

FIG. 2. Energy resolution of inorganic scintillators and of a HPGe detector for the detection of $662 \mathrm{keV}$ gamma photons. The energy resolution is defined as the full width at half maximum of total energy peak in scintillation pulse height spectra divided by the mean energy of that peak.

full-energy peak in the energy spectrum measured by a scintillation detector.

The nonproportionality of scintillators is attributed to radiationless recombination of electron-hole pairs with a recombination rate that increases with the ionization density. ${ }^{2,6,21-25}$ This process together with an ionization density that changes along an electron track and with primary electron energy causes the deterioration of the energy resolution. To avoid the recombination losses, charge carriers should be effectively transferred from the primary track to luminescence centers. The faster the charge carriers escape the volume of high ionization density shown in Fig. 1, in which quenching occurs, the higher the probability of converting carriers into optical photons. An important factor determining the rate at which carriers leave this volume is the carrier diffusion coefficient. ${ }^{2,6,23}$ A high diffusion coefficient contributes to a more rapid transport of electrons, holes and excitons to regions further from the track where the radiationless recombination rate does not depend on ionization density.

In this paper the dependence of $\mathrm{LaBr}_{3}$ nonproportionality on temperature and $\mathrm{Ce}^{3+}$ concentration has been studied. For $\mathrm{LaBr}_{3}$ with $0.2 \%, 5 \%$, and $30 \%$ of $\mathrm{Ce}^{3+}$, the nonproportional response is determined at $80 \mathrm{~K}, 300 \mathrm{~K}$, and $450 \mathrm{~K}$ and as a function of photon energy (photon-nPR) and as a function of electron energy (electron-nPR). Scintillation yield and energy resolution were measured in the energy range from $10.5 \mathrm{keV}$ to $100 \mathrm{keV}$ and at $662 \mathrm{keV}$. A specific model will be presented able to describe the electron-nPR results, and the degree of electron-nPR will be introduced and determined. Its dependence on temperature and concentration will be compared with our model estimate of the mobility for thermalized carriers in wide band gap semiconductors.

\section{EXPERIMENTAL METHODS}

To record scintillation pulse height spectra as a function of temperature, ${\mathrm{a} \mathrm{LaBr}_{3}}_{3}$ Ce sample was fixed at the bottom of a parabolic-like stainless steel cup covered with a reflective Al-foil, mounted onto the cold finger of a liquid nitrogen bath cryostat. The cup directs the scintillation light through a quartz window towards a photomultiplier tube (PMT) situated outside the cryostat chamber. The Hamamatsu R6231-100 PMT at $-680 \mathrm{~V}$ bias voltage remained at room temperature and observes about $20 \%$ of the emitted scintillation light. To collect as much of the PMT output charge pulse as possible, the shaping time of an Ortec 672 spectroscopic amplifier was set at $10 \mu \mathrm{s}$. The temperature of the sample was controlled by two thermocouples attached to different parts of the sample holder. The yield of the scintillator will be expressed by the number of photoelectrons created in the PMT per $\mathrm{MeV}\left(\mathrm{N}_{\text {phe }}{ }^{\mathrm{PMT}} / \mathrm{MeV}\right)$ of absorbed gamma or $\mathrm{X}$-ray photon energy. The energy resolution $R$ of a peak in the pulse height spectrum at energy $E$ is defined as the ratio of the full width at half maximum $\Delta E$ of that peak to the energy $E$, and it will be expressed as a percentage value.

To measure X-ray pulse height spectra at many finely spaced energy values between $10.5 \mathrm{keV}$ and $100 \mathrm{keV}$, experiments were carried out at the X-1 beam line at the Hamburger Synchrotronstrahlungslabor (HASYLAB) synchrotron radiation facility in Hamburg, Germany. A highly monochromatic pencil X-ray beam in the energy range $10.5-100 \mathrm{keV}$ was used as an excitation source. A tunable double Bragg reflection monochromator using a Si[511] set of silicon crystals providing an X-ray resolution of $1 \mathrm{eV}$ at $10.5 \mathrm{keV}$ rising to $20 \mathrm{eV}$ at $100 \mathrm{keV}$ was used to select the $\mathrm{X}$ ray energies. A sketch of the experimental set-up can be found in Ref. 26. The beam spot size was set by a pair of precision stepper-driven slits, positioned immediately in front of the cryostat chamber. For all measurements, a slit size of $50 \times 50 \mu \mathrm{m}^{2}$ was used.

A dense sampling of data performed around the lanthanum K-electron binding energy $E_{K L a}=38.925 \mathrm{keV}$ was done in order to apply the K-dip spectroscopy method. ${ }^{27}$ This method allows to derive the response of $\mathrm{LaBr}_{3}$ : $\mathrm{Ce}$ to photoelectrons down to energies as low as $100 \mathrm{eV}$. The method is briefly described as follows. An X-ray with energy $E_{X}$ that photoelectrically interacts with the lanthanum K-shell leads to the creation of a photoelectron with energy $E_{e}$ and a hole in the lanthanum $K$-shell,

$$
E_{e}=E_{X}-E_{K L a}
$$

The hole relaxes to the ground state with the emission of a cascade of secondary X-ray fluorescence photons and/or Auger electrons. The response of a scintillator is then equivalent to the sum of two main interaction products: (1) the 
K-shell photo electron response and (2) the response from the electrons emitted due to the sequence of processes following relaxation of the hole in the $\mathrm{K}$-shell, the so-called $\mathrm{K}$ cascade response. Our strategy is to employ X-ray energies just above $E_{K L a}$. The $\mathrm{K}$-cascade response is assumed to be independent from the original $\mathrm{X}$-ray energy. This response is found by tuning the X-ray energy to just above $E_{K L a}$. By subtracting the $\mathrm{K}$-cascade response from the total $\mathrm{X}$-ray response, we are left with the response in photoelectrons from the K-shell photoelectron alone with energy $E_{e}$. The $\mathrm{K}$-electron-nPR curve is then obtained from the number $N_{\text {phe }}^{P M T} / \mathrm{MeV}$ at the energy of the K-photoelectron divided by the number $N_{\text {phe }}^{P M T} / \mathrm{MeV}$ measured at $662 \mathrm{keV}$.

\section{RESULTS}

The photon nonproportional response (photon-nPR) written as $f_{p h}(E)$ is defined as the number of photoelectrons $N_{p h e}^{P M T} / \mathrm{MeV}$ of absorbed energy observed at energy $E$ divided by the number $N_{p h e}^{P M T} / \mathrm{MeV}$ observed at $E=662 \mathrm{keV}$ energy. $f_{p h}(E)$ is expressed as a percentage value. For an ideal proportional scintillator, it is $100 \%$ at all energies. Figure 3 shows $f_{p h}(E)$ for $\mathrm{LaBr}_{3}$ doped with $0.2 \%, 5 \%$, and $30 \% \mathrm{Ce}^{3+}$ studied at $80 \mathrm{~K}, 300 \mathrm{~K}$, and $450 \mathrm{~K}$. The shape of the $f_{p h}(E)$ curve depends not only on the temperature as was reported before, ${ }^{22}$ but here we found that it also depends on $\mathrm{Ce}^{3+}$ concentration.

As a figure of merit the degree of photon-nPR $\sigma_{p h}$ will be used. It has been defined following ideas in ${ }^{28-30}$

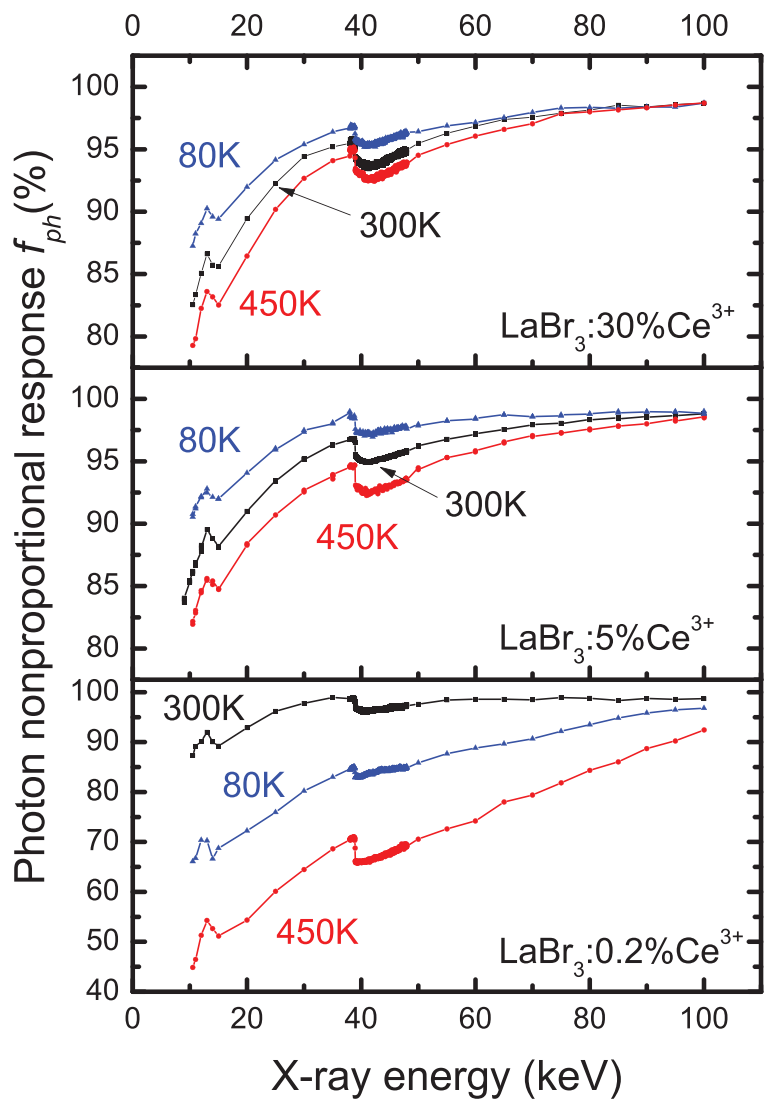

FIG. 3. Photon nonproportional response of $\mathrm{LaBr}_{3}$ doped with $0.2 \%, 5 \%$, and $30 \% \mathrm{Ce}^{3+}$ as a function of X-ray or gamma photon energy at $80 \mathrm{~K}$, $300 \mathrm{~K}$, and $450 \mathrm{~K}$.
TABLE I. Degree (in \%) of $\mathrm{LaBr}_{3}:$ Ce photon-nPR $\sigma_{p h}$ in the energy range from $E_{\text {min }}=10.5 \mathrm{keV}$ to $E_{\text {max }}=662 \mathrm{keV}$.

\begin{tabular}{lccc}
\hline \hline & \multicolumn{3}{c}{ Temperature, $\mathrm{K}$} \\
\cline { 2 - 4 } $\mathrm{Ce}^{3+}$ concentration (\%) & 80 & 300 & 450 \\
\hline 0.2 & 3.31 & 0.95 & 6.98 \\
5 & 0.78 & 1.07 & 1.43 \\
30 & 1.09 & 1.22 & 1.37 \\
\hline \hline
\end{tabular}

$$
\sigma_{p h}=\frac{1}{\left(E_{\max }-E_{\min }\right)} \int_{E_{\min }}^{E_{\max }}\left|f_{p h}\left(E_{\max }\right)-f_{p h}(E)\right| d E,
$$

where $E_{\text {max }}=662 \mathrm{keV}, E_{\text {min }}=10.5 \mathrm{keV}$, and $f_{p h}\left(E_{\text {max }}\right)$ is set equal to $100 \%$. $\sigma_{p h}$ for $\mathrm{LaBr}_{3}$ at different temperatures and $\mathrm{Ce}^{3+}$ concentrations obtained from the results in Fig. 3 are listed in Table I. For $\mathrm{LaBr}_{3}: 5 \% \mathrm{Ce}$ and $\mathrm{LaBr}_{3}: 30 \% \mathrm{Ce} \sigma_{p h}$ increases with temperature. The behavior is different for $\mathrm{LaBr}_{3}: 0.2 \% \mathrm{Ce}$ where the lowest value for $\sigma_{p h}$ is observed at $300 \mathrm{~K}$. The smallest $\sigma_{p h}$ is measured for $\mathrm{LaBr}_{3}: 5 \% \mathrm{Ce}$ at $80 \mathrm{~K}$.

The energy resolution $R(E)$ of $\mathrm{LaBr}_{3}$ doped with $0.2 \%$, $5 \%$, and $30 \% \mathrm{Ce}^{3+}$ at $80 \mathrm{~K}, 300 \mathrm{~K}$, and $450 \mathrm{~K}$ is presented in Fig. 4. The overall pattern is consistent with the pattern of $\sigma_{p h}$. At a given energy for both $\mathrm{LaBr}_{3}$ doped with $5 \%$ and $30 \% \mathrm{Ce}^{3+}$ the best energy resolution is obtained at $80 \mathrm{~K}$ and the worst at $450 \mathrm{~K}$. $\mathrm{LaBr}_{3}: 0.2 \% \mathrm{Ce}$ shows the best resolution at $300 \mathrm{~K}$ where $\sigma_{p h}$ is minimal. Figure 4 shows that at $80 \mathrm{~K}$,

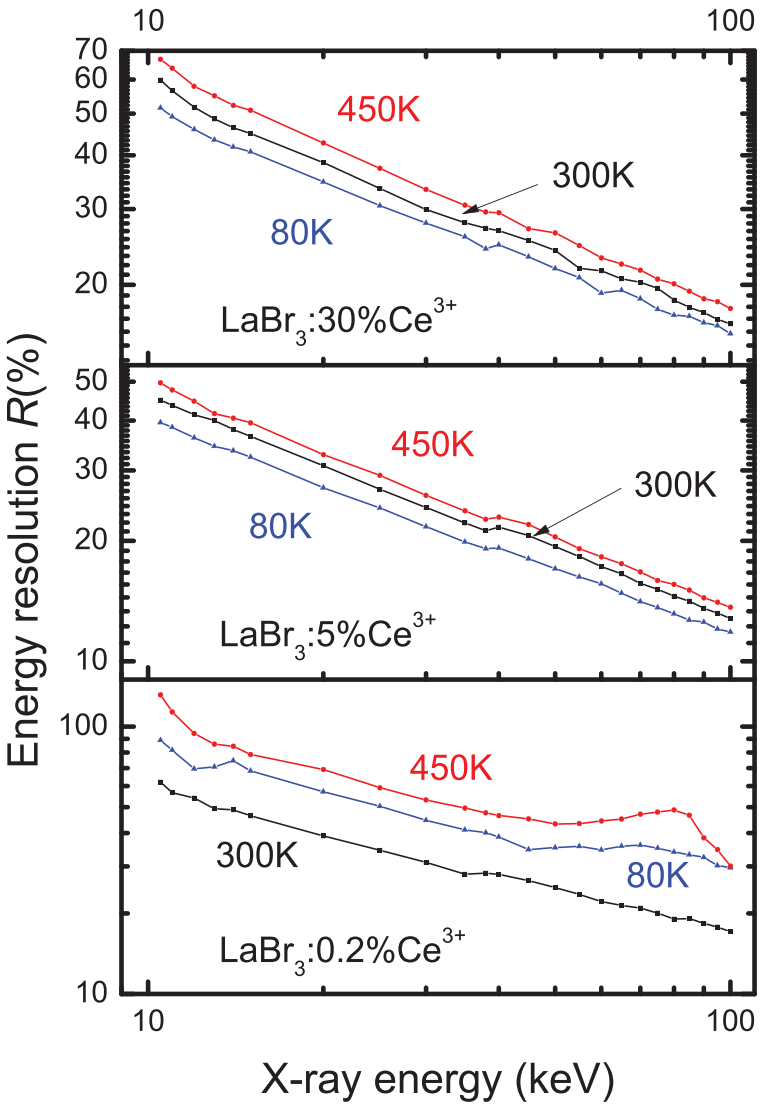

FIG. 4. Energy resolution of $\mathrm{LaBr}_{3}$ doped with $0.2 \%, 5 \%$, and $30 \% \mathrm{Ce}^{3+}$ as a function of X-ray energy at $80 \mathrm{~K}, 300 \mathrm{~K}$, and $450 \mathrm{~K}$. 
the already outstanding room temperature energy resolution of $\mathrm{LaBr}_{3}$ doped with 5 and $30 \% \mathrm{Ce}^{3+}$ can be improved even further. To confirm the dependence of $R$ on temperature and concentration, pulse height spectra were recorded using ${ }^{137} \mathrm{Cs} 662 \mathrm{keV}$ gamma radiation.

The energy resolution $\Delta E / E$ of a scintillator is determined by

$$
\begin{aligned}
\left(\frac{\Delta E}{E}\right)^{2} & =R^{2}=R_{M}^{2}(T)+R_{s c}^{2}(T) \\
& =(2.35)^{2} \frac{1+v(M)}{N_{p h e}^{P M T}(T)}+R_{s c}^{2}(T)
\end{aligned}
$$

where $v(M)$ is the variance in the PMT gain, $N_{p h e}^{P M T}$ is the number of photoelectrons that are produced by the interaction of scintillation photons with the PMT photocathode and are multiplied on the first dynode, ${ }^{31}$ and $R_{s c}$ is given by

$$
R_{s c}^{2}(T)=R_{n P R}^{2}(T)+R_{t r}^{2}(T)+R_{i n h}^{2}(T),
$$

where $R_{n P R}(T)$ is a contribution from nonproportionality, $R_{t r}(T)$ is the so-called transport resolution, and $R_{i n h}(T)$ is a contribution from inhomogeneity of the scintillation crystal. It is assumed that all contributions are independent from each other.

To measure the temperature dependence of the $\mathrm{LaBr}_{3}: \mathrm{Ce}$ energy resolution the parabolic-like cup covered with reflective $\mathrm{Al}$ foil was used. This configuration of the experimental set-up results in the collection of only about $20 \%$ of the emitted scintillation photons which then increases the statistical contribution $R_{M}(T)$.

Figure 5 shows the measured $R(T), R_{M}(T)$ calculated from the measured $N_{p h e}^{P M T}$, and $R_{s c}(T)$ obtained with Eq. (4) for $\mathrm{LaBr}_{3}: 0.2 \% \mathrm{Ce}$. The parabolas through the data are drawn to guide the eye. $R_{M}(T)$ is small and $R(T)$ is almost entirely determined by $R_{s c}(T)$. The resolution is lowest at room temperature. This pattern is consistent with the pattern of $\sigma_{p h}$ in Table I where a larger $\sigma_{p h}$ results in poorer energy resolution which confirms a relationship between energy resolution and nonproportionality.

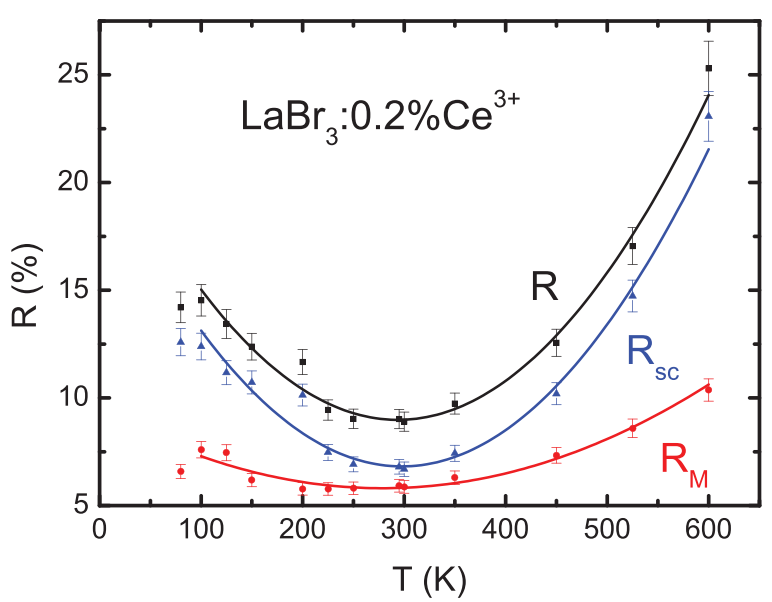

FIG. 5. The separate contributions to the total energy resolution of $\mathrm{LaBr}_{3}: 0.2 \% \mathrm{Ce}$ at $662 \mathrm{keV}$ as a function of temperature.

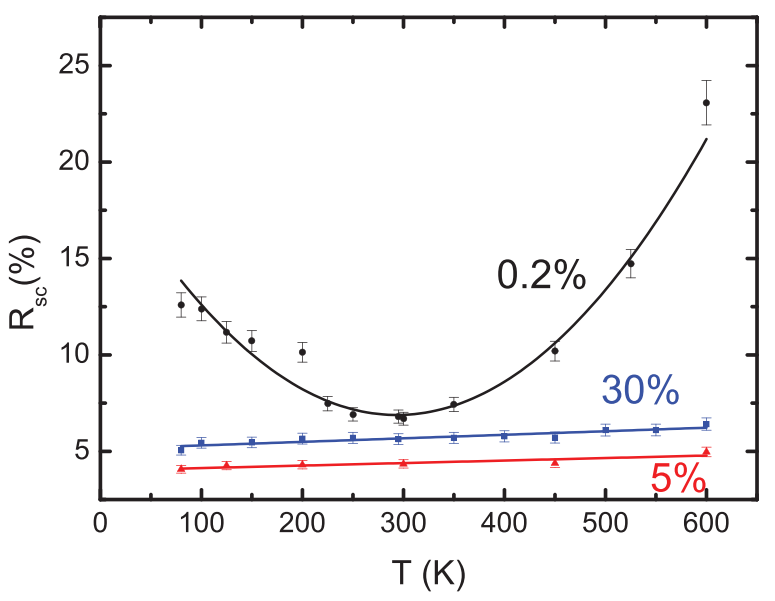

FIG. 6. The $R_{s c}$ contribution to the energy resolution at $662 \mathrm{keV}$ of $0.2 \%$, $5 \%$, and $30 \% \mathrm{Ce}$-doped $\mathrm{LaBr}_{3}$ as a function of temperature.

The contribution $R_{s c}(T)$ to the energy resolution at $662 \mathrm{keV}$ is shown in Fig. 6 for $0.2 \%, 5 \%$, and $30 \%$ Ce-doped $\mathrm{LaBr}_{3} . \mathrm{LaBr}_{3}: 0.2 \% \mathrm{Ce}$ shows a minimum at room temperature. In contrast, $\mathrm{LaBr}_{3}$ with $5 \%$ and $30 \% \mathrm{Ce}^{3+}$ exhibits a linear decrease of the $R_{s c}(T)$ with decreasing temperature. Lower values of $R_{s c}(T)$ correlate with lower values of $\sigma_{p h}$.

Using K-dip spectroscopy, we derived the K-photoelectron-nPR curves $f_{e}(E)$ for $\mathrm{LaBr}_{3}$ doped with $0.2 \%, 5 \%$, and $30 \% \mathrm{Ce}^{3+}$ at $80 \mathrm{~K}, 300 \mathrm{~K}$, and $450 \mathrm{~K}$ which are shown in Fig. 7.

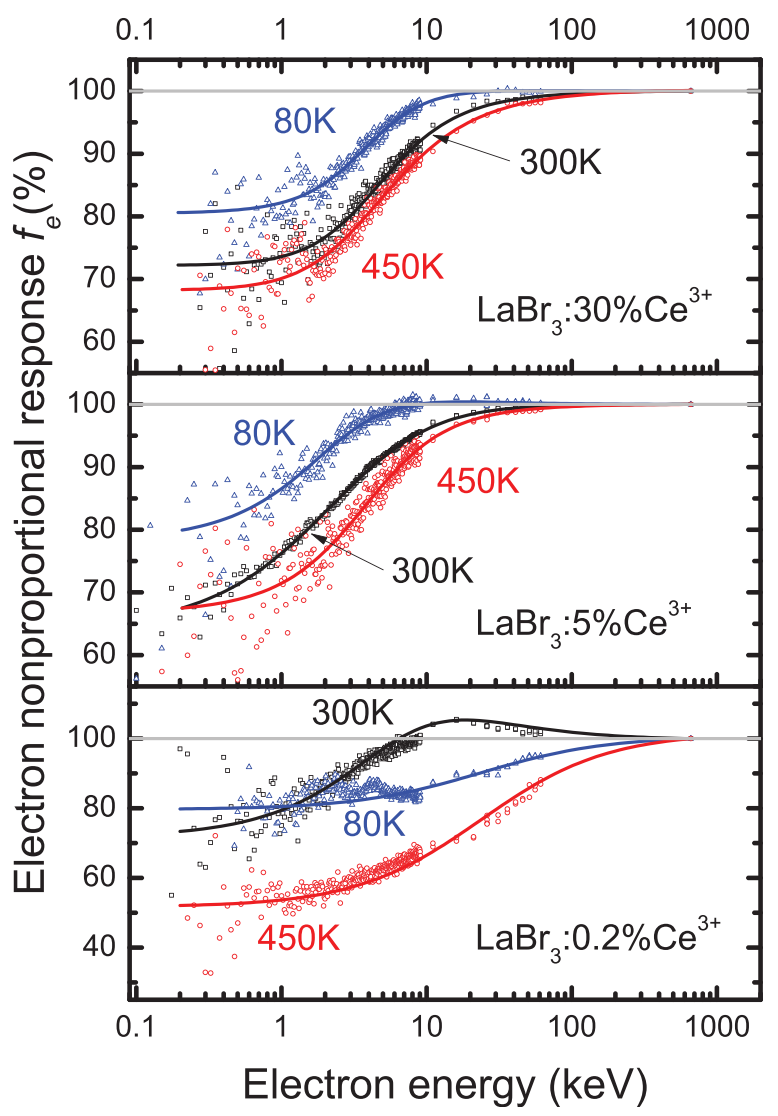

FIG. 7. K-photoelectron nonproportional response of $\mathrm{LaBr}_{3}$ doped with $0.2 \%, 5 \%$, and $30 \% \mathrm{Ce}^{3+}$ as a function of X-ray or gamma photon energy at $80 \mathrm{~K}, 300 \mathrm{~K}$, and $450 \mathrm{~K}$. The solid curves are drawn to guide the eye. 


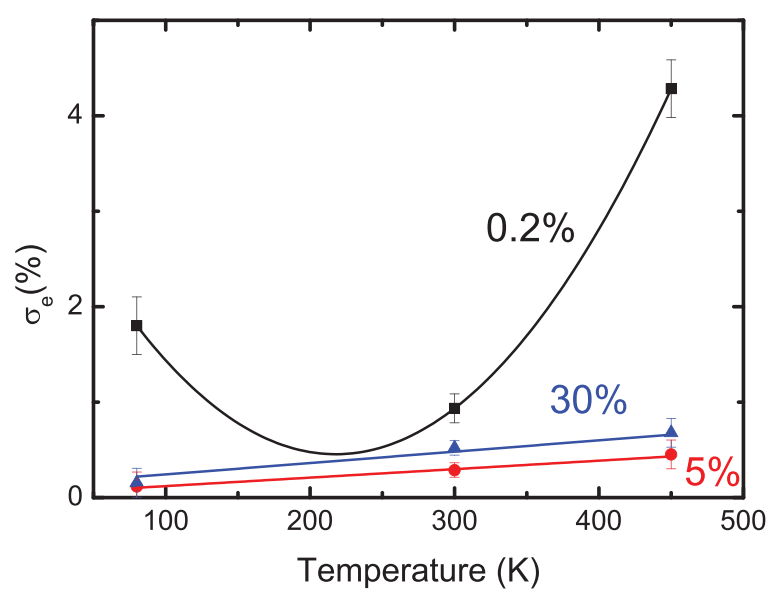

FIG. 8. Degree of $\mathrm{LaBr}_{3}$ electron-nPR $\sigma_{e}$ versus temperature and $\mathrm{Ce}^{3+}$ concentration. The solid curves are drawn to guide the eye.

TABLE II. Degree (in \%) of the $\mathrm{LaBr}_{3}$ :Ce electron-nPR $\sigma_{e}$ in the energy range from $E_{\text {min }}=0.2 \mathrm{keV}$ to $E_{\max }=662 \mathrm{keV}$.

\begin{tabular}{lccc}
\hline \hline & \multicolumn{3}{c}{ Temperature, K } \\
\cline { 2 - 4 } $\mathrm{Ce}^{3+}$ concentration (\%) & 80 & 300 & 450 \\
\hline 0.2 & 1.80 & 0.93 & 4.28 \\
5 & 0.12 & 0.29 & 0.45 \\
30 & 0.16 & 0.52 & 0.68 \\
\hline \hline
\end{tabular}

$\sigma_{e}$ is defined analogous to the degree of photon-nPR and is determined using Eq. (2) by integrating over the energy range from $E_{\text {min }}=0.2 \mathrm{keV}$ to $E_{\text {max }}=662 \mathrm{keV}$. For a perfectly proportional scintillator, the value of $\sigma_{e}$ is zero, and the scintillator with a lower value of $\sigma_{e}$ is considered to be more proportional.

$\sigma_{e}$ versus $T$ and $\mathrm{Ce}^{3+}$ concentration is shown in Fig. 8 and in Table II. It behaves similar to $\sigma_{p h}$. The only difference is that $\sigma_{e}$ of $\mathrm{LaBr}_{3}: 0.2 \% \mathrm{Ce}$ at $300 \mathrm{~K}$ shows a higher value of $0.93 \%$ compared to $0.29 \%$ for $\mathrm{LaBr}_{3}: 5 \% \mathrm{Ce}$ and $0.52 \%$ for $\mathrm{LaBr}_{3}: 30 \%$ Ce. Linear extrapolation of $\sigma_{e}$ for $\mathrm{LaBr}_{3}: 5 \% \mathrm{Ce}$ and $\mathrm{LaBr}_{3}: 30 \% \mathrm{Ce}$ suggests that $\sigma_{e}$ for both concentrations reach zero at a temperature close to the absolute zero. This means, that an almost perfect proportional response would be obtained for $5 \%$ and for $30 \%$ Ce-doped $\mathrm{LaBr}_{3}$ crystals.

\section{DISCUSSION}

Using synchrotron irradiation, the photon-nPR $f_{p h}(E)$ and energy resolution $R$ of $\mathrm{LaBr}_{3}: \mathrm{Ce}$ scintillation crystals doped with $0.2 \%, 5 \%$, and $30 \%$ of $\mathrm{Ce}^{3+}$ were studied at $80 \mathrm{~K}, 300 \mathrm{~K}$, and $450 \mathrm{~K}$. Results of these experiments were shown in Figs. 3 and 4 and in Table I. $f_{p h}(E)$ and $\sigma_{p h}$ are characteristics of the gamma photon response of a scintillator, however, the response to energetic electrons is more fundamental. If $f_{e}(E)$ is known and when the process of ionization track creation can be simulated, the shape of $f_{p h}(E)$ over the entire energy range can be calculated ${ }^{32,33}$ by Monte-Carlo techniques. The actual value of $f_{p h}(E)$ at energy $E$ is then a weighted average of several values of $f_{e}(E)$ at lower energies. ${ }^{20}$ Using the electron-nPR function $f_{e}(E)$ then provides a better starting point to understand nonproportionality then using the photon-nPR function. ${ }^{20,34}$ Using the Kdip spectroscopy method $f_{e}(E)$ shown in Fig. 7 was derived from the $f_{p h}(E)$ and Table II was calculated using Eqs. (2) and (7). Figure 8 shows $\sigma_{e}$ of $\mathrm{LaBr}_{3}$ versus temperature and $\mathrm{Ce}^{3+}$ concentration, and this figure is the most important outcome of the performed experiments and calculations. In the following discussion, we will concentrate on better understanding of the results in Fig. 8 by using ideas on carrier mobility from semiconductor physics and apply them to the processes that occur inside the ionization track in scintillators.

There are several models proposed in the recent literature to explain the origin of nonproportionality. 2,6,21,29,35,36 It is attributed to radiationless electron-hole pair recombination in the regions of a high concentration $n(x)$ of charge carriers along the ionization track as shown in Fig. 1.

Assuming cylindrical shape of high ionization density volume $^{1}$ along the track of the primary energetic electron as shown in Fig. 1, the concentration of the ionized charge carriers $n(x)$ is given by

$$
n(x)=\frac{1}{\pi r^{2} E_{e-h}}\left(-\frac{d E}{d x}\right),
$$

where $r$ is the radius of the high ionization density volume shown in Fig. 1 and $E_{e-h}$ is the average energy required to create a free electron-free hole pair in the scintillator. 5,37

$n(x)$ increases with smaller energy $E$ of the track creating primary electron. ${ }^{38}$ This leads to a larger radiationless electron hole recombination rate which forms the basis of increasing nonproportionality with smaller gamma or X-ray photon or primary electron energy.

An overview of the current models on nonproportionality was presented by Moses et al. ${ }^{24}$ The basis of all those models is the competition between two opposing processes shown in Fig. 1: (1) quenching due to radiationless electron hole recombination inside the volume of high ionization density along the track, and (2) diffusion of the charge carriers from the point of creation towards a volume of lower ionization density. The faster the charge carriers escape the volume of high ionization density in which quenching occurs and reach luminescence centers, the higher the probability of converting the energy of the carriers into optical photons. An important factor determining the rate at which carriers leave this volume is the carrier diffusion coefficient. ${ }^{2,6,23}$ Another very important parameter is concentration of luminescence or trapping centers inside the high ionization density volume. At high concentration of $\mathrm{Ce}^{3+}$ in $\mathrm{LaBr}_{3}$ essential part of the charge carriers can be promptly removed from the diffusionquenching process. According to Bizarri and Dorenbos, ${ }^{39}$ carriers can be sequentially captured by $\mathrm{Ce}^{3+}$ or form selftrapped excitons (STE) which transfer their energy to $\mathrm{Ce}^{3+}$ through thermally activated migration or directly. These effects can lead to a significant difference of quenching probability at low Ce concentration, $0.2 \%$, and at high concentrations $5 \%$ and $30 \%$. The minimum in $\sigma_{e}$ for $\mathrm{LaBr}_{3}: 0.2 \% \mathrm{Ce}^{3+}$ in Fig. 8 at room temperature suggests a minimum in the loss 
processes at room temperature that within the above theory should correspond with a maximum in charge carrier mobility. According to theory of charge carrier transport in wide band gap semiconductors, mobility indeed strongly depends on temperature. ${ }^{40,41}$ Here, we will employ that theory in order to understand the results for $\mathrm{LaBr}_{3}: 0.2 \% \mathrm{Ce}^{3+}$ in Fig. 8. The theory is for thermalized charge carriers and we therefore assume that all charge carriers are thermalized instantly $^{42,43}$ after creation in the ionization track.

An increase of carrier mobility with temperature decrease is due to a reduced phonon interaction rate. Emission of optical phonons is the main mechanism responsible for carrier scattering by the lattice. $\mathrm{LaBr}_{3}$ does not show any piezoelectric properties. That means that piezoelectric mode scattering caused by the electric field associated with acoustical phonons can also be ignored in our calculations. Lattice scattering due to optical phonons is independent on the carrier concentration.

Using standard theory on diffusion of thermalized charge carriers and one obtains for the radial concentration change with time

$$
\left.\frac{\partial n(r, t)}{\partial t}\right|_{\text {diffusion }}=\mu(T) \cdot k T \cdot \nabla^{2} n(r, t),
$$

where $\mu(T)$ is the mobility of the charge carriers, $k$ is the Boltzmann constant and $T$ is the effective temperature of the charge carriers. The transport of charge carriers becomes faster when carrier mobility and temperature increases. An increase of carrier mobility with temperature decrease can be caused by a reduced phonon interaction rate. Emission of optical phonons is the main mechanism responsible for carrier scattering by the lattice which is independent on the carrier concentration. $^{40}$

The main lattice scattering mechanism is due to the interaction of carriers with the longitudinal-optical phonons. According to Ref. 40, the optical Hall lattice mobility for electrons can be calculated from

$$
\mu_{L_{o p t}}=\frac{e}{2 \alpha \omega_{0} m^{*}}\left(\exp \left(\frac{\hbar \omega_{0}}{k T}\right)-1\right),
$$

where $\alpha$ is the polaron coupling constant given by

$$
\alpha=\left(\frac{1}{\varepsilon_{\infty}}-\frac{1}{\varepsilon}\right) \sqrt{\frac{m * E_{H}}{m_{e} \hbar \omega_{0}}} .
$$

For $\mathrm{LaBr}_{3}$, the high frequency and the static dielectric constants are $\varepsilon_{\infty} \approx 5$ and $\varepsilon \approx 10$, respectively; ${ }^{44}$ $E_{H}=13.595 \mathrm{eV}$ is the first ionization energy of the hydrogen atom; $\frac{m^{*}}{m_{e}}=1.323$ is the effective electron mass divided by the electron mass; ${ }^{29} \hbar \omega_{0}=23.7 \mathrm{meV}$ is the energy of the longitudinal-optical phonon in $\mathrm{LaBr}_{3}$. ${ }^{44}$

An increase of carrier mobility with temperature increase in our model can only be caused by ionized impurity scattering, ${ }^{41}$ which according to $\operatorname{Ziman}^{41}$ is given by

$$
\mu_{i}=\frac{2^{7 / 2}\left(\varepsilon \varepsilon_{0}\right)^{2}(k T)^{3 / 2}}{\pi^{3 / 2} z^{2} e^{3}\left(m^{*}\right)^{1 / 2} N_{i}} \cdot F(3 k T),
$$

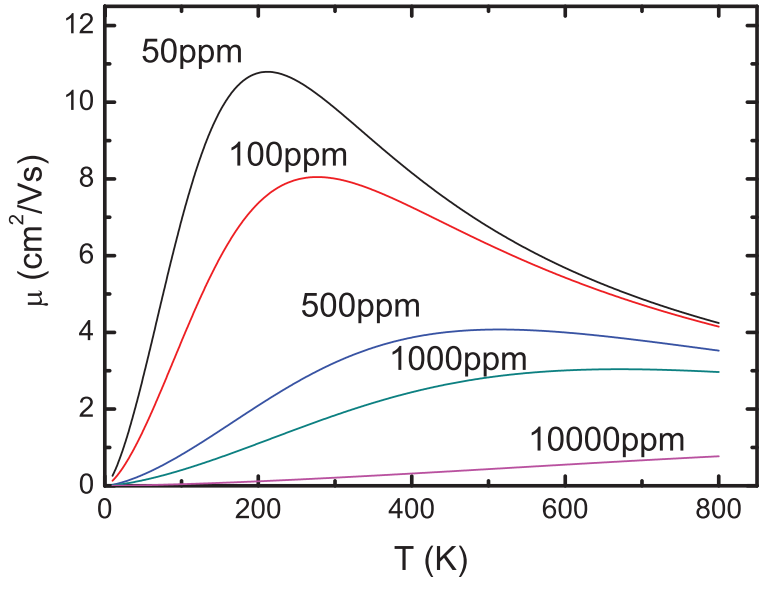

FIG. 9. Calculated mobility of electrons in $\mathrm{LaBr}_{3}$ versus temperature and ionized impurity concentration.

where $z$ is the effective charge of the impurity with concentration $N_{i}, \varepsilon_{0}$ is the vacuum permittivity and $F(3 k T)$ is the averaged Coulomb screening factor, ${ }^{41}$ and for our range of temperatures $F(3 k T) \approx 1$. By ionized impurity scattering not compensated charged defects or impurities are considered. Neutral impurity scattering mechanism is not taken into consideration because it's mobility is at least two orders of magnitude higher than one due to ionized impurity scattering. ${ }^{41}$

The overall electron mobility $\mu(T)$ can be obtained from

$$
\mu(T)=\left(\frac{1}{\mu_{L}(T)}+\frac{1}{\mu_{i}(T)}\right)^{-1} .
$$

Figure 9 shows the mobility calculated with Eq. (10) for different concentrations of ionized impurity scattering centers with $\mathrm{z}=1$ in $\mathrm{LaBr}_{3}$. At impurity concentration of $100 \mathrm{ppm}$, the maximum of the carrier mobility is slightly below room temperature. Therefore with our model, an impurity concentration of $100 \mathrm{ppm}$ is needed to match well with the minimum of $R_{s c}(T)$ of $\mathrm{LaBr}_{3}: 0.2 \% \mathrm{Ce}$ at $300 \mathrm{~K}$ in Fig. 5 and of $\sigma_{e}$ in Fig. 8.

Equations (7)-(10) pertain to a given density of carriers in the conduction or valence band. The calculations do not incorporate any carrier trapping ${ }^{39}$ and also it was assumed that all charge carriers are thermalized instantly ${ }^{42,43}$ after creation in the ionization track. However, recent theoretical studies $^{35,45}$ suggest that also non-thermalized carriers play an important role in carrier and phonon transport in scintillators. One should therefore interpret the results in Fig. 9 as qualitative.

Lattice and impurity scattering mechanisms are expected to be more important at low $\mathrm{Ce}^{3+}$ concentration due to the longer distance carriers need to travel before they can reach $\mathrm{Ce}^{3+}$ where they can recombine radiatively. The concentration of $\mathrm{Ce}^{3+}$ in $\mathrm{LaBr}_{3}: 0.2 \% \mathrm{Ce}$ is $4.2 \cdot 10^{18} \mathrm{~cm}^{-3}$. At 5 and $30 \% \mathrm{Ce}^{3+}$ concentration the carrier density $n(x)$ is 1.05 . $10^{20} \mathrm{~cm}^{-3}$ and $6.3 \cdot 10^{20} \mathrm{~cm}^{-3}$ which is of the same order of magnitude as the concentration of recombination centers, and a high mobility of charge carriers needed to escape the dense ionization region becomes of less importance. Carriers can be trapped instantly after ionization and the trapping rate by 
$\mathrm{Ce}^{3+}$ starts to dominate over the quenching rate and the escape rate. This can explain the better $\sigma_{e}$ shown in Fig. 8 for 5 and $30 \%$ Ce concentration.

Recently, a significant improvement of $\mathrm{LaBr}_{3}: 5 \% \mathrm{Ce}$ scintillation yield proportionality and energy resolution by means of $\mathrm{Ca}^{2+}, \mathrm{Sr}^{2+}$, or $\mathrm{Ba}^{2+}$ co-doping was demonstrated ${ }^{46,47}$ Incorporation of $100 \mathrm{ppm}$ of $\mathrm{Sr}^{2+}$, a charged impurity with $\mathrm{z}=1$, resulted in a large improvement of the photon-nPR that brings energy resolution of $\mathrm{LaBr}_{3}$ to $2 \%$ at $662 \mathrm{keV}$ which is almost at the theoretical limit shown in Fig. 2. The model proposed in this work does not fully predict such type of result for high concentrations of $5 \% \mathrm{Ce}^{3+}$ in $\mathrm{LaBr}_{3}$, but at the same time, it does give clear indication that few hundred ppm of charged impurities can strongly influence carrier mobilities and lead to improvement of proportionality.

\section{CONCLUSION}

The shape of the photon- and electron-nPR curves of $\mathrm{LaBr}_{3}: \mathrm{Ce}$ depends on temperature. For $5 \%$ and $30 \% \mathrm{Ce}^{3+}$ concentration, $\mathrm{LaBr}_{3}$ shows better proportionality and energy resolution when temperature decreases. This improvement means that at a low temperature even better energy resolution can be achieved with $\mathrm{LaBr}_{3}$ scintillation detector compared to the already outstanding $2.75 \%$ measured at room temperature.

The temperature dependence of the photon- and electron-nPRs of $\mathrm{LaBr}_{3}: 0.2 \% \mathrm{Ce}$ is different. The most proportional response was measured at $300 \mathrm{~K}$. At $80 \mathrm{~K}$ and $450 \mathrm{~K}$, the photon- and electron-nPR curves deviate strongly from the linear response. This leads to a significant deterioration of the energy resolution both at $80 \mathrm{~K}$ and $450 \mathrm{~K}$.

Despite the limitations of the theoretical model that was used, the obtained results suggest that a significant factor determining the nonproportionality of $\mathrm{LaBr}_{3}: 0.2 \% \mathrm{Ce}$ is the mobility of charge carriers. The higher the carrier mobility and diffusion coefficient the lower the degree of electronnPR, which leads to improved energy resolution. Semiconductor detectors based on HPGe with excellent energy resolution of $0.3 \%$ besides different statistics have a much higher mobility of charge carriers $\sim 40000 \mathrm{~cm}^{2} / \mathrm{Vs}$ compared to $\sim 8 \mathrm{~cm}^{2} / \mathrm{Vs}$ calculated for $\mathrm{LaBr}_{3}: 0.2 \%$ Ce with $100 \mathrm{ppm}$ ionized impurity concentration. For 5\% and 30\% concentrations direct trapping by the recombination centers starts do dominate and a high mobility of charge carriers becomes of less importance.

Summarizing the results of the performed measurements and calculations and bearing in mind that carrier mobility in semiconductor detectors is high, we conclude that the "ultimate energy resolution" should be sought in scintillation materials with high carrier mobility and high charge carrier capture efficiency.

\section{ACKNOWLEDGMENTS}

The research leading to these results has received funding from the Netherlands Technology Foundation (STW), Saint Gobain, crystals and detectors division, Nemours,
France, and by the European Community's Seventh Framework Programme (FP7/2007-2013) under Grant Agreement No. 226716. We thank the scientists and technicians of the X-1 beamline at the Hamburger Synhrotronstrahlungslabor (HASY-LAB) synchrotron radiation facilities for their assistance. The authors want to acknowledge Conny Hansson, Johannes van der Biezen and Alan Owens from the European Space Agency (ESTEC) for their assistance with the experiment and sharing some of the beam time at X-1.

${ }^{1}$ A. N. Vasil'ev, IEEE Trans. Nucl. Sci. 55, 1054 (2008).

${ }^{2}$ R. T. Williams, J. Q. Grim, Q. Li, K. B. Ucer, and W. W. Moses, Phys. Status Solidi B 248, 426 (2011).

${ }^{3}$ M. Kirm et al., Phys. Rev. B 79, 233103 (2009).

${ }^{4}$ V. Nagirnyi, S. Dolgov, R. Grigonis, M. Kirm, L. L. Nagornaya, F. Savikhin, V. Sirutkaitis, S. Vielhauer, and A. Vasil'ev, IEEE Trans. Nucl. Sci. 57, 1182 (2010).

${ }^{5}$ P. A. Rodnyi, Physical Processes in Inorganic Scintillators (CRC Press, NY, 1997).

${ }^{6}$ Q. Li, J. Q. Grim, R. T. Williams, G. Bizarri, and W. W. Moses, J. Appl. Phys. 109, 123716 (2011).

${ }^{7}$ E. V. D. van Loef, P. Dorenbos, C. W. E. van Eijk, K. Kramer, and H. U. Gudel, Appl. Phys. Lett. 79, 1573 (2001).

${ }^{8}$ E. V. D. van Loef, P. Dorenbos, C. W. E. van Eijk, K. W. Kramer, and H. U. Gudel, Phys. Rev. B 68, 045108 (2003).

${ }^{9}$ N. J. Cherepy et al., Appl. Phys. Lett 92, 083508 (2008).

${ }^{10}$ G. Bizarri, E. D. Bourret-Courchesne, Z. Yan, and S. E. Derenzo, IEEE Trans. Nucl. Sci. 58, 3403 (2011).

${ }^{11}$ L. Onsager, Phys. Rev. 54, 554 (1938).

${ }^{12}$ M. Luntz, Phys. Rev. B 4, 2857 (1971).

${ }^{13}$ G. Shwetha and V. Kanchana, Phys. Rev. B 86, 115209 (2012).

${ }^{14}$ D. Singh, Phys. Rev. B 82, 155145 (2010).

${ }^{15}$ M. A. Verdier, P. C. F. Di Stefano, P. Nadeau, C. Behan, M. Clavel, and C. Dujardin, Phys. Rev. B 84, 214306 (2011).

${ }^{16}$ A. Canning, A. Chaudhry, R. Boutchko, and N. Granbech-Jensen, Phys. Rev. B 83, 125115 (2011).

${ }^{17}$ D. Aberg, B. Sadigh, and P. Erhart, Phys. Rev. B 85, 125134 (2012).

${ }^{18}$ P. Dorenbos, IEEE Trans. Nucl. Sci. 57, 1162 (2010).

${ }^{19}$ P. Dorenbos, J. T. M. de Haas, and C. W. E. van Eijk, IEEE Trans. Nucl. Sci. 42, 2190 (1995).

${ }^{20}$ W. W. Moses, S. A. Payne, W. S. Choong, G. Hull, and B. W. Reutter, IEEE Trans. Nucl. Sci. 55, 1049 (2008).

${ }^{21}$ G. Bizarri, W. W. Moses, J. Singh, A. N. Vasil'ev, and R. T. Williams, J. Appl. Phys. 105, 044507 (2009).

${ }^{22}$ I. V. Khodyuk, M. S. Alekhin, J. T. M. de Haas, and P. Dorenbos, Nucl. Instrum. Methods A 642, 75 (2011).

${ }^{23}$ Q. Li, J. Q. Grim, R. T. Williams, G. Bizarri, and W. W. Moses, Nucl. Instrum. Methods A 652, 288 (2011).

${ }^{24}$ W. W. Moses, G. Bizarri, R. T. Williams, and S. A. Payne, IEEE Trans. Nucl. Sci. 59, 2038 (2012).

${ }^{25}$ R. B. Murray and A. Meyer, Phys. Rev. 122, 815 (1961).

${ }^{26}$ A. Owens, A. J. J. Bos, S. Brandenburg, P. Dorenbos, W. Drozdowski, R. W. Ostendorf, F. Quarati, A. Webb, and E. Welter, Nucl. Instrum. Methods A 574, 158 (2007).

${ }^{27}$ I. V. Khodyuk, J. T. M. de Haas, and P. Dorenbos, IEEE Trans. Nucl. Sci. 57, 1175 (2010).

${ }^{28} \mathrm{P}$. Dorenbos, Nucl. Instrum. Methods A 486, 208 (2002).

${ }^{29}$ W. Setyawan, R. M. Gaume, R. S. Feigelson, and S. Curtarolo, IEEE Trans. Nucl. Sci. 56, 2989 (2009).

${ }^{30}$ I. V. Khodyuk and P. Dorenbos, IEEE Trans. Nucl. Sci. 59, 3320 (2012).

${ }^{31}$ J. T. M. de Haas and P. Dorenbos, IEEE Trans. Nucl. Sci. 58, 1290 (2011).

${ }^{32}$ B. D. Rooney and J. D. Valentine, IEEE Trans. Nucl. Sci. 44, 509 (1997).

${ }^{33}$ M. M. Terekhov, R. L. Aptekar, D. D. Frederiks, S. V. Golenetskii, V. N. Il'inskii, and E. P. Mazets, in Gamma-Ray Bursts, Pts 1 and 2 (Amer Inst Physics, Melville, 1998), p. 894.

${ }^{34}$ E. V. D. van Loef, W. Mengesha, J. D. Valentine, P. Dorenbos, and C. W. E. van Eijk, IEEE Trans. Nucl. Sci. 50, 155 (2003).

${ }^{35}$ A. Kozorezov, J. K. Wigmore, and A. Owens, J. Appl. Phys. 112, 053709 (2012). 
${ }^{36}$ S. A. Payne, W. W. Moses, S. Sheets, L. Ahle, N. J. Cherepy, B. Sturm, S. Dazeley, G. Bizarri, and C. Woon-Seng, IEEE Trans. Nucl. Sci. 58, 3392 (2011).

${ }^{37}$ P. A. Rodnyi, P. Dorenbos, and C. W. E. van Eijk, Phys. Status Solidi B 187, 15 (1995).

${ }^{38}$ G. F. Knoll, Radiation Detection and Measurement (John Wiley \& Sons, Inc., the United States of America, 1979).

${ }^{39}$ G. Bizarri and P. Dorenbos, Phys. Rev. B 75, 184302 (2007).

${ }^{40} \mathrm{~K}$. Ellmer, A. Klein, and B. Rech, Transparent Conductive Zinc Oxide (Springer, Berlin, 2008).

${ }^{41}$ J. M. Ziman, Electrons and phonons (The Clarendon Press, Oxford, 1960).
${ }^{42}$ A. N. Belsky et al., J. Electron Spectrosc. Relat. Phenom. 79, 147 (1996).

${ }^{43}$ Q. Li, J. Q. Grim, K. B. Ucer, A. Burger, G. Bizarri, W. W. Moses, and R. T. Williams, Phys. Status Solidi (RRL) 6, 346 (2012).

${ }^{44}$ B. Liu, M. Gu, Z. Qi, X. Liu, S. Huang, and C. Ni, Phys. Rev. B 76, 064307 (2007).

${ }^{45}$ R. Kirkin, V. V. Mikhailin, and A. N. Vasil'ev, IEEE Trans. Nucl. Sci. 59, 2057 (2012)

${ }^{46}$ M. S. Alekhin, J. T. M. de Haas, I. V. Khodyuk, K. W. Kraemer, P. Menge, V. Ouspenski, and P. Dorenbos, Appl. Phys. Lett. 102, 161915 (2013).

${ }^{47}$ M. S. Alekhin, D. A. Biner, K. W. Kraemer, and P. Dorenbos, J. Appl. Phys. 113, 224904 (2013). 